\title{
Electronic heat current fluctuations in a quantum dot
}

\author{
A. Crépieux ${ }^{1}$ \\ 1 Aix Marseille Univ, Université de Toulon, CNRS, CPT, Marseille, France
}

\begin{abstract}
The fluctuations of the heat current in a quantum dot coupled to electron reservoirs are calculated at finite frequency, voltage and temperature using the nonequilibrium Green function technique. The non-symmetrized heat noise is expressed as an integral on energy containing four contributions, each of which includes transmission amplitudes, electron-hole pair distribution functions and energy difference factors. The effect of the asymmetry of the couplings between the quantum dot and the reservoirs is studied. Features of the heat noise are highlighted and discussed for an equilibrium and an out-of-equilibrium quantum dot. In the latter case and within the high transmission limit, the heat noise is closely related to the radiative power spectrum, leading to an out-of-equilibrium Planck's law. Proposals for the measurement of the heat noise are discussed.
\end{abstract}

\section{INTRODUCTION}

In quantum devices, the heat fluctuates over time for several reasons: the first one is related to the presence of thermal agitation at finite temperature, the second one to the fact that the device interacts with its electromagnetic environment by emitting or absorbing energy via phonons or photons, and the third one to the probabilistic nature of particle transfer in quantum systems. The characterization of these heat fluctuations provides a variety of information on energy dissipation ${ }^{1}$, the presence of finite coherence and entanglement in open quantum systems ${ }^{2}$, and the higher-order cumulants of charge counting statistics ${ }^{3}$. In addition, they reveal features that are not visible in the charge noise ${ }^{4}$ such as the signature of a crossover from Coulomb blockade to Kondo physics in energy fluctuations ${ }^{5}$. In the case of ondemand single-electron sources, the heat fluctuates while the charge emission is noiseless 6 . So far, only temperature fluctuations ${ }^{7}$, related to energy fluctuations $\sqrt{61899}$, have been measured 10 , but there are several proposals for the measurement of heat fluctuations ${ }^{8|9| 11 \mid 12}$. With the fast progress of heat measurement techniques in nanosystems $\frac{13}{16}$, it can be expected that this will be possible in the foreseeable future. Heat transport in quantum devices is in itself well controlled ${ }^{17}$, notably with the experimental confirmation $\frac{18}{18}$ of the existence of a thermal conductance quantum $\frac{19}{2}$, and the evidence of the heat Coulomb blockade effect ${ }^{20}$.

The issues raised by these studies are also of fundamental interest. The question of the generalization of the fluctuation-dissipation theorem to heat transport has been addressed 21 23, as well as the verification of the fluctuation theorem $12 \mid 24 \sqrt{28}$, which is a microscopic extension of the second law of thermodynamics. The statistics of heat exchange in a driven open quantum system have been studied ${ }^{29}$ as well as the statistics of work for a two-level system in the presence of dissipation 30 . Among the theoretical approaches used to study the heat fluctuations in quantum devices, one can cite the Landauer-Büttiker formalism ${ }^{3132}$, nonequilibrium Schwinger-Keldysh Green function technique $e^{3 / 33 / 35}$, circuit theory $^{27}$, Tomonaga-Luttinger liquid theory ${ }^{36137}$, generalized Lindblad master equations $2 \mid 12128$, meanfield method coupled to Hartree-Fock approximation ${ }^{38}$, Boltzmann-Langevin approach $\underline{\underline{9}}$, and inchworm quantum Monte Carlo method ${ }^{5}$. The systems in question are either molecular junctions ${ }^{3438}$, quantum wires ${ }^{3136 / 39}$, mesoscopic constrictions ${ }^{21}$, quantum dots 1112 |27/35/40|41/43 , double quantum dots 144 , or qubits 45 . In these works, the generating function for the heat full-counting statistics has been determined $1 / 3|5| 8|24| 33|39| 43 \mid 47$, and the zero-frequency heat noise has been calculated $\sqrt{3 / 5131 / 36 / 38 / 40 \mid 47 / 49}$ as well as the symmetrized finite-frequency heat noise $\mathrm{e}^{21|22| 32|34| 35}$. The non-symmetrized finite-frequency heat noise has also been calculated for a quantum dot (QD) but only for symmetrical couplings between the QD and the electron reservoirs ${ }^{41}$. The objective of the present work is twofold: first to generalize the calculation of non-symmetrized finite-frequency heat noise to the case of asymmetrical couplings, which can differ by a factor of up to ten 2 , by looking at both auto-correlators and cross-correlators, and second to highlight the main features of the heat noise spectrum in a QD. Only the electronic contribution to the heat noise is considered in this work.

The paper is organized as follows: the model and results are presented in Sec. II, the equilibrium and outof-equilibrium heat noises are, respectively, discussed in Secs. III and IV, and the conclusion is given in Sec. V.

\section{MODEL AND RESULTS}

The standard Hamiltonian describing a non-interacting QD connected to left $(\mathrm{L})$ and right $(\mathrm{R})$ reservoirs of electrons is the Anderson Hamiltonian,

$$
\begin{aligned}
\mathcal{H}= & \sum_{\alpha=L, R} \sum_{k \in \alpha} \varepsilon_{\alpha k} c_{\alpha k}^{\dagger} c_{\alpha k}+\varepsilon_{0} d^{\dagger} d \\
& +\sum_{\alpha=L, R} \sum_{k \in \alpha}\left(V_{\alpha k} c_{\alpha k}^{\dagger} d+\text { h.c. }\right)
\end{aligned}
$$

where $c_{\alpha k}^{\dagger}\left(d^{\dagger}\right), c_{\alpha k}(d)$ are the creation and annihilation operators associated with the reservoir $\alpha$ (respectively the $\mathrm{QD}$ ). The energies $\varepsilon_{\alpha k}, \varepsilon_{0}$ and $V_{\alpha k}$ are respectively the energy of the electrons in the reservoir 
$\alpha$, the discrete energy level of the QD, and the hopping integral between the reservoirs and the QD. The retarded Green function associated with the QD connected to the reservoirs is given in the flat wide-band limit by
$G^{r}(\varepsilon)=\left(\varepsilon-\varepsilon_{0}+i\left(\Gamma_{L}+\Gamma_{R}\right) / 2\right)^{-1}$, where $\Gamma_{\alpha}=2 \pi \rho_{\alpha}\left|V_{\alpha}\right|^{2}$ is the coupling between the QD and the reservoir $\alpha$ assuming that the density of states $\rho_{\alpha}$ and $V_{\alpha} \equiv V_{\alpha k}$ are energy independent.

\begin{tabular}{|l||c|c|c|c|}
\hline$M_{\alpha \beta}^{\gamma \delta}(\varepsilon, \omega)$ & $\gamma=\delta=L$ & $\gamma=\delta=R$ & $\gamma=L, \delta=R$ & $\gamma=R, \delta=L$ \\
\hline \hline$\alpha=L$ & $\mid \mathcal{E}_{L}(\varepsilon-\omega) t_{L L}(\varepsilon)$ & $\mathcal{E}_{L}^{2}\left(\varepsilon-\frac{\omega}{2}\right)$ & $\mid \mathcal{E}_{L}(\varepsilon)$ & $\mathcal{E}_{L}(\varepsilon-\omega)$ \\
$\beta=L$ & $+\mathcal{E}_{L}(\varepsilon) t_{L L}^{*}(\varepsilon-\omega)$ & $\times \mathcal{T}_{L R}(\varepsilon) \mathcal{T}_{L R}(\varepsilon-\omega)$ & $-\left.\mathcal{E}_{L}\left(\varepsilon-\frac{\omega}{2}\right) t_{L L}(\varepsilon)\right|^{2}$ & $-\left.\mathcal{E}_{L}\left(\varepsilon-\frac{\omega}{2}\right) t_{L L}(\varepsilon-\omega)\right|^{2}$ \\
& $-\left.\mathcal{E}_{L}\left(\varepsilon-\frac{\omega}{2}\right) t_{L L}(\varepsilon) t_{L L}^{*}(\varepsilon-\omega)\right|^{2}$ & & $\times \mathcal{T}_{L R}(\varepsilon-\omega)$ & $\times \mathcal{T}_{L R}(\varepsilon)$ \\
\hline$\alpha=R$ & $\mathcal{E}_{R}^{2}\left(\varepsilon-\frac{\omega}{2}\right)$ & $\mid \mathcal{E}_{R}(\varepsilon-\omega) t_{R R}(\varepsilon)$ & $\mid \mathcal{E}_{R}(\varepsilon-\omega)$ & $\mid \mathcal{E}_{R}(\varepsilon)$ \\
$\beta=R$ & $\times \mathcal{T}_{L R}(\varepsilon) \mathcal{T}_{L R}(\varepsilon-\omega)$ & $+\mathcal{E}_{R}(\varepsilon) t_{R R}^{*}(\varepsilon-\omega)$ & $-\left.\mathcal{E}_{R}\left(\varepsilon-\frac{\omega}{2}\right) t_{R R}(\varepsilon-\omega)\right|^{2}$ & $-\left.\mathcal{E}_{R}\left(\varepsilon-\frac{\omega}{2}\right) t_{R R}(\varepsilon)\right|^{2}$ \\
& & $-\left.\mathcal{E}_{R}\left(\varepsilon-\frac{\omega}{2}\right) t_{R R}(\varepsilon) t_{R R}^{*}(\varepsilon-\omega)\right|^{2}$ & $\times \mathcal{T}_{L R}(\varepsilon)$ & $\times \mathcal{T}_{L R}(\varepsilon-\omega)$ \\
\hline$\alpha=L$ & $\mathcal{E}_{R}\left(\varepsilon-\frac{\omega}{2}\right) t_{L R}(\varepsilon) t_{L R}^{*}(\varepsilon-\omega)$ & $\mathcal{E}_{L}\left(\varepsilon-\frac{\omega}{2}\right) t_{L R}^{*}(\varepsilon) t_{L R}(\varepsilon-\omega)$ & {$\left[\mathcal{E}_{L}(\varepsilon) t_{L L}(\varepsilon)\right.$} & {$\left[\mathcal{E}_{L}(\varepsilon-\omega) t_{L L}^{*}(\varepsilon-\omega)\right.$} \\
$\beta=R$ & $\times\left[\mathcal{E}_{L}\left(\varepsilon-\frac{\omega}{2}\right) t_{L L}^{*}(\varepsilon) t_{L L}(\varepsilon-\omega)\right.$ & $\times\left[\mathcal{E}_{R}\left(\varepsilon-\frac{\omega}{2}\right) t_{R R}(\varepsilon) t_{R R}^{*}(\varepsilon-\omega)\right.$ & $\left.-\mathcal{E}_{L}\left(\varepsilon-\frac{\omega}{2}\right) \mathcal{T}_{L L}(\varepsilon)\right]$ & $\left.-\mathcal{E}_{L}\left(\varepsilon-\frac{\omega}{2}\right) \mathcal{T}_{L L}(\varepsilon-\omega)\right]$ \\
& $-\mathcal{E}_{L}(\varepsilon-\omega) t_{L L}^{*}(\varepsilon)$ & $-\mathcal{E}_{R}(\varepsilon-\omega) t_{R R}(\varepsilon)$ & $\times\left[\mathcal{E}_{R}(\varepsilon-\omega) t_{R R}(\varepsilon-\omega)\right.$ & $\times\left[\mathcal{E}_{R}(\varepsilon) t_{R R}^{*}(\varepsilon)\right.$ \\
& $\left.-\mathcal{E}_{L}(\varepsilon) t_{L L}(\varepsilon-\omega)\right]$ & $\left.-\mathcal{E}_{R}(\varepsilon) t_{R R}^{*}(\varepsilon-\omega)\right]$ & $\left.-\mathcal{E}_{R}\left(\varepsilon-\frac{\omega}{2}\right) \mathcal{T}_{R R}(\varepsilon-\omega)\right]$ & $\left.-\mathcal{E}_{R}\left(\varepsilon-\frac{\omega}{2}\right) \mathcal{T}_{R R}(\varepsilon)\right]$ \\
\hline$\alpha=R$ & $\mathcal{E}_{R}\left(\varepsilon-\frac{\omega}{2}\right) t_{L R}^{*}(\varepsilon) t_{L R}(\varepsilon-\omega)$ & $\mathcal{E}_{L}\left(\varepsilon-\frac{\omega}{2}\right) t_{L R}(\varepsilon) t_{L R}^{*}(\varepsilon-\omega)$ & {$\left[\mathcal{E}_{L}(\varepsilon) t_{L L}^{*}(\varepsilon)\right.$} & {$\left[\mathcal{E}_{L}(\varepsilon-\omega) t_{L L}(\varepsilon-\omega)\right.$} \\
$\beta=L$ & $\times\left[\mathcal{E}_{L}\left(\varepsilon-\frac{\omega}{2}\right) t_{L L}(\varepsilon) t_{L L}^{*}(\varepsilon-\omega)\right.$ & $\times\left[\mathcal{E}_{R}\left(\varepsilon-\frac{\omega}{2}\right) t_{R R}^{*}(\varepsilon) t_{R R}(\varepsilon-\omega)\right.$ & $\left.-\mathcal{E}_{L}\left(\varepsilon-\frac{\omega}{2}\right) \mathcal{T}_{L L}(\varepsilon)\right]$ & $\left.-\mathcal{E}_{L}\left(\varepsilon-\frac{\omega}{2}\right) \mathcal{T}_{L L}(\varepsilon-\omega)\right]$ \\
& $-\mathcal{E}_{L}(\varepsilon-\omega) t_{L L}(\varepsilon)$ & $-\mathcal{E}_{R}(\varepsilon-\omega) t_{R R}^{*}(\varepsilon)$ & $\times\left[\mathcal{E}_{R}(\varepsilon-\omega) t_{R R}^{*}(\varepsilon-\omega)\right.$ & $\times\left[\mathcal{E}_{R}(\varepsilon) t_{R R}(\varepsilon)\right.$ \\
& $\left.-\mathcal{E}_{L}(\varepsilon) t_{L L}^{*}(\varepsilon-\omega)\right]$ & $\left.-\mathcal{E}_{R}(\varepsilon) t_{R R}(\varepsilon-\omega)\right]$ & $\left.-\mathcal{E}_{R}\left(\varepsilon-\frac{\omega}{2}\right) \mathcal{T}_{R R}(\varepsilon-\omega)\right]$ & $\left.-\mathcal{E}_{R}\left(\varepsilon-\frac{\omega}{2}\right) \mathcal{T}_{R R}(\varepsilon)\right]$ \\
\hline
\end{tabular}

TABLE I: Expressions of matrix elements $M_{\alpha \beta}^{\gamma \delta}(\varepsilon, \omega)$ appearing in the finite-frequency heat noise of Eq. 2 , setting $\hbar=1$, where $t_{\alpha \beta}(\varepsilon)=i \sqrt{\Gamma_{\alpha} \Gamma_{\beta}} G^{r}(\varepsilon)$ is the transmission amplitude, $\mathcal{T}_{\alpha \beta}(\varepsilon)=\left|t_{\alpha \beta}(\varepsilon)\right|^{2}$ is the transmission coefficient, and $\mathcal{E}_{\alpha}(\varepsilon)=\varepsilon-\mu_{\alpha}$ is the difference between the energy $\varepsilon$ of the particle and the chemical potential in the reservoir $\alpha$.

The heat noise is defined as the Fourier transform of the non-symmetrized correlator of heat currents at two different times: $\mathcal{S}_{\alpha \beta}^{\text {heat }}(\omega)=\int_{-\infty}^{\infty}\left\langle\Delta J_{\alpha}(t) \Delta J_{\beta}(0)\right\rangle e^{-i \omega t} d t$, where $\Delta J_{\alpha}(t)=J_{\alpha}(t)-\left\langle J_{\alpha}\right\rangle$. The heat current operator is given by $\sqrt[50]{53} J_{\alpha}(t)=-\dot{\mathcal{H}}_{\alpha}+\mu_{\alpha} \dot{N}_{\alpha}$, where $\mathcal{H}_{\alpha}=\sum_{k \in \alpha} \varepsilon_{\alpha k} c_{\alpha k}^{\dagger} c_{\alpha k}$ is the Hamiltonian of the uncoupled reservoir $\alpha$ and $N_{\alpha}=\sum_{k \in \alpha} c_{\alpha k}^{\dagger} c_{\alpha k}$ is the operator number of electrons in the reservoir $\alpha$. The calculation of the non-symmetrized finite-frequency heat noise is performed using the nonequilibrium Green function technique. It gives 54

$\mathcal{S}_{\alpha \beta}^{\text {heat }}(\omega)=\frac{1}{h} \sum_{\gamma, \delta=L, R} \int_{-\infty}^{\infty} d \varepsilon M_{\alpha \beta}^{\gamma \delta}(\varepsilon, \omega) f_{\gamma}^{e}(\varepsilon) f_{\delta}^{h}(\varepsilon-\hbar \omega)$

where $f_{\gamma}^{e}(\varepsilon)=\left(1+\exp \left(\left(\varepsilon-\mu_{\gamma}\right) / k_{B} T_{\gamma}\right)\right)^{-1}$ and $f_{\delta}^{h}(\varepsilon)=$ $1-f_{\delta}^{e}(\varepsilon)$ are the Fermi-Dirac distributions for the electrons in the reservoir $\gamma$ and the holes in the reservoir $\delta$. $\mu_{\gamma}$ and $T_{\gamma}$ are respectively the chemical potential and the temperature in the reservoir $\gamma$. The matrix elements
$M_{\alpha \beta}^{\gamma \delta}(\varepsilon, \omega)$ are listed in Table I. The relative importance of the four terms in Eq. (2) varies according to the experimental conditions: they all have an equal weight at equilibrium while the term $M_{\alpha \beta}^{L R}(\varepsilon, \omega)$ dominates out-ofequilibrium. The result of Eq. (2) is applicable to any frequency $\omega$, temperatures $T_{L, R}$, voltage $V$ and couplings $\Gamma_{L, R}$. It generalizes the results of Ref. 41 to arbitrary couplings between the QD and the reservoirs. The expressions of the elements for the matrix $M$ reduce to the ones entering in the expression of the charge noise $\mathcal{S}_{\alpha \beta}^{\text {charge }}(\omega)$ of Refs. 55 and 56 provided that the factor $\mathcal{E}_{\alpha}(\varepsilon)$ is replaced by the value 1 . One notices that three of such factors enter into the expression of the heat noise: $\mathcal{E}_{\alpha}(\varepsilon)$, the energy of the electron in the reservoir $\alpha$, $\mathcal{E}_{\alpha}(\varepsilon-\hbar \omega)$, the energy of the hole in the reservoir $\alpha$, and $\mathcal{E}_{\alpha}(\varepsilon-\hbar \omega / 2)$, the average energy of the electron-hole pair in the reservoir $\alpha$. These factors are related to the energy exchanged with the electromagnetic environment surrounding the QD during the various transfer processes contributing to the heat noise. These processes are 10 in number (for $\mathcal{S}_{L L}^{\text {heat }}(\omega)$, see Fig. 1) and involve transfer of 


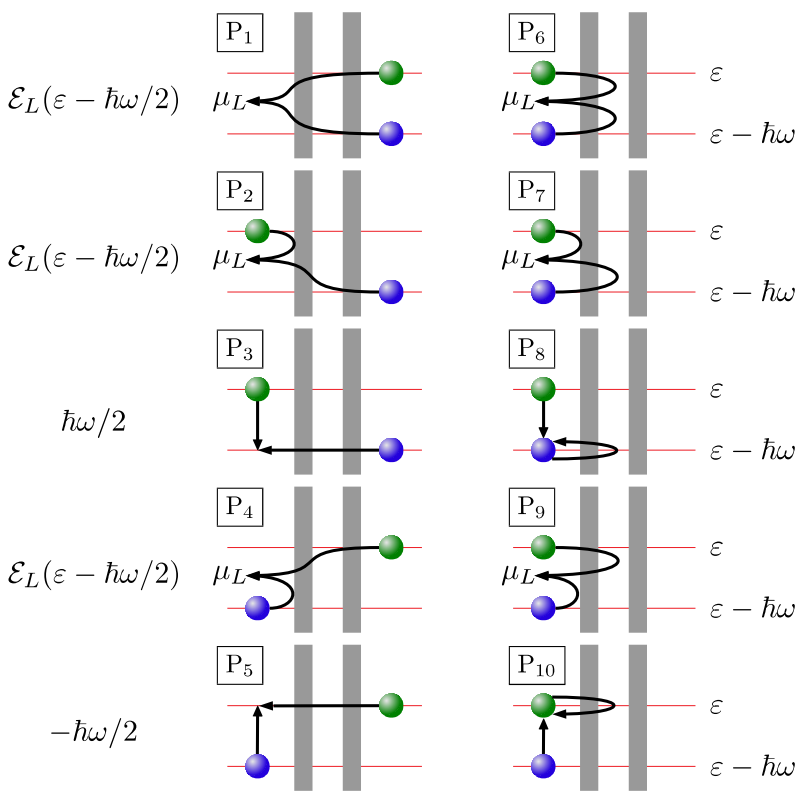

FIG. 1: Illustration of the 10 processes contributing to the heat noise in the left reservoir attached to the QD. The green (blue) spheres represent an electron (hole) of energy $\varepsilon(\varepsilon-\hbar \omega)$. The energy released for each process is indicated on the left side.

electron-hole pairs through the QD. Depending on the initial location of the electron and the hole, the number of possible processes differs. When the electron and the hole are both located in the right reservoir, there is only one process: $\mathrm{P}_{1}$. When the electron is located in the left (right) reservoir and the hole in the right (left) reservoir, there are two processes: $\mathrm{P}_{2}$ and $\mathrm{P}_{3}\left(\mathrm{P}_{4}\right.$ and $\left.\mathrm{P}_{5}\right)$. Finally, when the electron and the hole are both initially located in the left reservoir, there are five processes: from $\mathrm{P}_{5}$ to $\mathrm{P}_{10}$. To calculate the contribution of each of these sets of processes, one must take the quantum superposition of the processes having the same initial state ${ }^{54}$. Using this simple rule, one recognizes the expression of $\mathcal{S}_{L L}^{\text {heat }}(\omega)$ of Table I. It highlights the fact that the energy exchanged with the electromagnetic environment during the processes is the average energy of the electron-hole pair, i.e., either $\mathcal{E}_{L}(\varepsilon-\hbar \omega / 2)$, when the electron and the hole both make an excursion in the central part of the $\mathrm{QD}$, or $\pm \hbar \omega / 2$, when only one of the two particles makes the excursion. This is the case for the processes $\mathrm{P}_{3}, \mathrm{P}_{5}$, $\mathrm{P}_{8}$ and $\mathrm{P}_{10}$, which have the particularity to contribute to the heat noise whereas they do not contribute to the charge noise ${ }^{55}$.

\section{EQUILIBRIUM HEAT NOISE}

Before exploiting the result of Eq. 22, one checks that it gives the expected behavior for heat noise within known limits. At zero frequency $\omega=0$, symmetrical couplings $\Gamma_{L, R}=\Gamma$ with $\mathcal{T}(\varepsilon)=\Gamma^{2} G^{r}(\varepsilon) G^{a}(\varepsilon)$, and using the op-
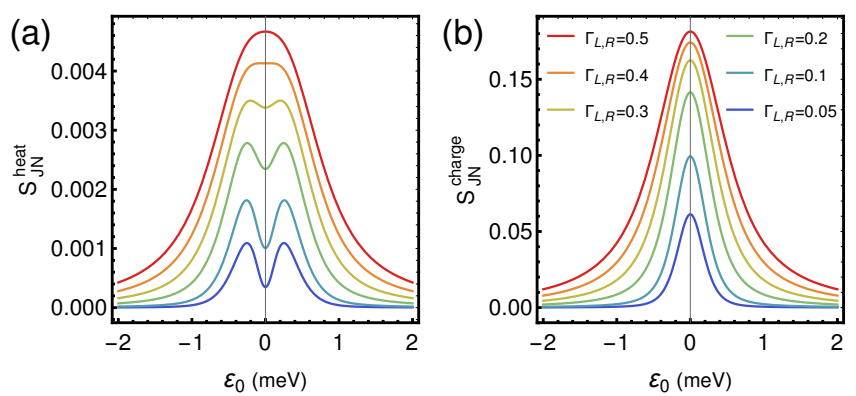

FIG. 2: Johnson-Nyquist (a) heat and (b) charge noises at equilibrium $\left(e V=\hbar \omega=0\right.$ and $\left.T_{L, R}=T\right)$ as a function of the QD level energy $\varepsilon_{0}$ at $k_{B} T=0.1 \mathrm{meV}$ (i.e., $T=1.16 \mathrm{~K}$ ) for several values of the couplings between the reservoirs and the QD taken symmetrical: $\Gamma_{L, R}=\Gamma$ (in meV).

tical theorem that holds for a non-interacting QD, which means that one has $t(\varepsilon)+t^{*}(\varepsilon)=2 \mathcal{T}(\varepsilon)$, Eq. (2) leads for the auto-correlators $(\alpha=\beta)$ to the expression $\underline{54}$

$$
\begin{aligned}
& \mathcal{S}_{\alpha \alpha}^{\text {heat }}(0)=\frac{1}{h} \int_{-\infty}^{\infty} d \varepsilon\left(\varepsilon-\mu_{\alpha}\right)^{2} \\
& \times\left[\mathcal{T}(\varepsilon)(1-\mathcal{T}(\varepsilon))\left(f_{\alpha}^{e}(\varepsilon)-f_{\bar{\alpha}}^{e}(\varepsilon)\right)^{2}\right. \\
& \left.+\mathcal{T}(\varepsilon)\left(f_{\alpha}^{e}(\varepsilon) f_{\alpha}^{h}(\varepsilon)+f_{\bar{\alpha}}^{e}(\varepsilon) f_{\bar{\alpha}}^{h}(\varepsilon)\right)\right]
\end{aligned}
$$

in agreement with the results of Refs. $\underline{436 / 43}$. The in$\operatorname{dex} \bar{\alpha}$ takes the value $R$ for $\alpha=L$ and the value $L$ for $\alpha=R$. The last line of Eq. (3) corresponds to the equilibrium heat noise $\mathcal{S}_{\text {JN }}^{\text {heat }}$ (Johnson-Nyquist), which can be expressed as a function of the thermal conductance $K_{\alpha}=\partial\left\langle J_{\alpha}\right\rangle / \partial T_{\alpha}$ by the relation $\mathcal{S}_{\mathrm{JN}}^{\text {heat }}=k_{B} T_{L}^{2} K_{L}+$ $k_{B} T_{R}^{2} K_{R}$, in perfect agreement with Refs $\frac{36 / 47 / 57}{3}$. One is reminded that the equilibrium charge noise $\mathcal{S}_{\text {JN }}^{\text {charge }}$ is related to the electrical conductance by the relation $\mathcal{S}_{\mathrm{JN}}^{\text {charge }}=k_{B} T_{L} G_{L}+k_{B} T_{R} G_{R}$, with $G_{\alpha}=e \partial\left\langle I_{\alpha}\right\rangle / \partial \mu_{\alpha}$, where $\left\langle I_{\alpha}\right\rangle$ is the electrical current associated to the reservoir $\alpha$. The Johnson-Nyquist heat and charge noises are displayed in Figs. 2(a) and (b) as a function of the QD energy level $\varepsilon_{0}$. Within a certain range of values for the coupling $\Gamma, \mathcal{S}_{\text {JN }}^{\text {heat }}$ shows a double-peak profile while a single one is observed in $\mathcal{S}_{\mathrm{JN}}^{\text {charge }}$. Indeed, at equilibrium, the charge fluctuations are maximal when the QD energy level is aligned with the chemical potentials, i.e. at $\varepsilon_{0}=0$ when $\mu_{L, R}=0$, since charge transfer does not cost energy. It results in a local minimum in the heat noise at $\varepsilon_{0}=0$. For increasing values of $\left|\varepsilon_{0}\right|$, the heat noise starts to increase because the charge transfer costs energy in this case. Then it finally decreases and converges to zero due to the fact that the probability for the charge to be transferred through the dot vanishes at high $\left|\varepsilon_{0}\right|$. When the two peaks in $\mathcal{S}_{\mathrm{JN}}^{\text {heat }}$ are present, their positions are $\varepsilon_{0} \approx \pm 2.5 k_{B} T$ at most ${ }^{54}$ in line with Ref. 58 where such a double peak structure has been predicted in the 
thermal conductance of a QD. The condition to have a double peak in $\mathcal{S}_{\text {JN }}^{\text {heat }}$ can be identified ${ }^{54}$. One finds that the condition is $\Gamma_{L}+\Gamma_{R} \lesssim 8 k_{B} T$. These results could be verified experimentally since at equilibrium the heat noise is proportional to the thermal conductance.

\section{OUT-OF-EQUILIBRIUM HEAT NOISE}

The heat noise is sensitive to the fact that the system is driven out-of-equilibrium either by applying a bias voltage, a temperature gradient, or by considering the noise at finite frequency. Figure 3 shows the profiles of heat and charge noises in the left reservoir as a function of the frequency at low temperature and fixed value of the voltage. For increasing values of the couplings $\Gamma_{L, R}$ (here taken symmetrical), the profile of the charge noise displayed in Fig. 3(b) changes until it vanishes for strong couplings since the transmission becomes perfect in this limit $\left(\mathcal{T}_{L R}(\varepsilon) \approx 1\right)$ which means that no fluctuation of charge current can occur: $\mathcal{S}_{L L}^{\text {charge }}(\omega)=0$. This is not the case for heat noise. On the contrary, one observes that the heat noise globally increases when the couplings increase until it reaches the curve of equation

$$
\mathcal{S}_{\alpha \alpha}^{\text {heat }}(\omega>0, T=0)=\frac{\hbar^{2} \omega^{2}}{4 h}(e V-\hbar \omega) \Theta(e V-\hbar \omega)
$$

derived in Ref. 54 in the zero temperature and perfect transmission limits, corresponding to the dashed black line in Fig. 3(a). Here, $\Theta$ is the Heaviside function. More generally the heat noise at perfect transmission is expressed as follows

$$
\begin{aligned}
\mathcal{S}_{\alpha \alpha}^{\text {heat }}(\omega)= & \frac{\hbar \omega}{h}\left(\frac{\hbar^{2} \omega^{2}}{6}+e^{2} V^{2}+2 e^{2} \mathcal{L} T^{2}\right) N(\hbar \omega) \\
& +\frac{\hbar^{2} \omega^{2}}{4 h} \sum_{ \pm}(\hbar \omega \pm e V) N(\hbar \omega \pm e V)
\end{aligned}
$$

with $N(\hbar \omega)$ the Bose-Einstein distribution function, and $\mathcal{L}=\pi^{2} k_{B}^{2} / 3 e^{2}$ the Lorenz number. This is a key result that generalizes the Planck's law for driven out-ofequilibrium quantum systems 59 . It gives $\mathcal{S}_{\alpha \alpha}^{\text {heat }}(\omega) \propto$ $\hbar^{3} \omega^{3} N(\hbar \omega)$ at zero voltage and low temperature, and Eq. (4) at positive voltage and zero temperature since one has $N_{T=0}(\varepsilon)=-\Theta(-\varepsilon)$. It means that the heat noise could be interpreted as the radiative power spectrum associated with the QD, opening the route to its measure. Thus, contrary to the charge noise, the heat noise does not vanish within the perfect transmission limit. It is related to the fact that external sources of energy are supplied to the system, by the applied voltage or the frequency of the measurement device, resulting in fluctuations of the heat current, except when $\hbar \omega>\mathrm{eV}$ since the system cannot deliver energy at a frequency higher than the voltage in the low-temperature limit ${ }^{64}$.

An additional distinctive feature between heat and charge noises is obtained for asymmetrical couplings (a)
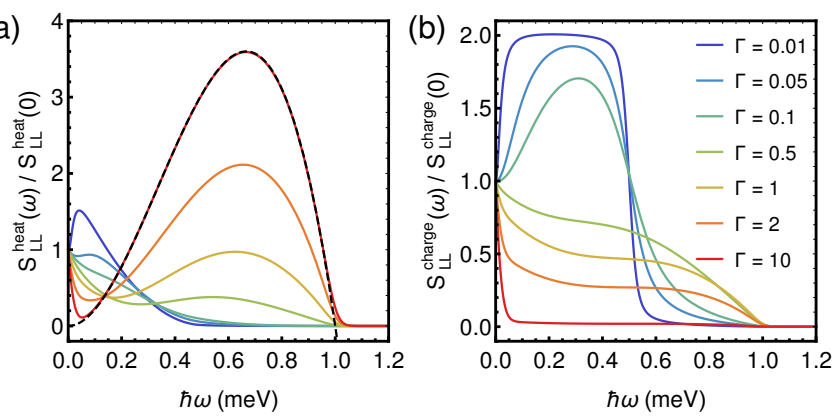

FIG. 3: Out-of-equilibrium (a) heat noise and (b) charge noise in the left reservoir as a function of frequency at $k_{B} T_{L, R}=$ $0.01 \mathrm{meV}, \varepsilon_{0}=0$, and $e V=1 \mathrm{meV}$ for different coupling values taken symmetrical: $\Gamma=\Gamma_{L, R}$ (in meV). The dashed black line in (a) corresponds to Eq. (4).

$\Gamma_{L} \neq \Gamma_{R}$. Figure 4(a) shows the heat noise in the left reservoir as a function of voltage for different values of the asymmetry factor $a=\Gamma_{L} / \Gamma_{R}$ at low temperature and strong couplings. One observes that the variation of $\mathcal{S}_{L L}^{\text {heat }}(\omega)$ changes from a linear variation with $e V$ at $a=1$ to a quadratic variation with $e V$ at $a \neq 1$. Therefore, the fact that heat noise varies linearly or quadratically with voltage could provide information on the asymmetry of couplings. Note that this asymmetry only manifests itself when the QD is in a nonequilibrium situation when $\mathrm{eV}$ and $\hbar \omega$ are both non-zero with the constraint $\hbar \omega<e V$. Such a linear/quadratic variation fits with the analytical expression, displayed by the dashed lines in Fig. 4(a), obtained for heat noise at zero temperature and perfect transmission 54

$$
\begin{aligned}
\mathcal{S}_{\alpha \alpha}^{\text {heat }}(\omega>0, T=0)= & \frac{\hbar \omega}{4 h}(e V-\hbar \omega) \Theta(e V-\hbar \omega) \\
& \times\left[\left(\frac{\Gamma_{\alpha}}{\Gamma_{\bar{\alpha}}}-1\right) e V+\hbar \omega\right]
\end{aligned}
$$

This result can be written alternatively under the form $\mathcal{S}_{\alpha \alpha}^{\text {heat }}(\omega)=\Delta U \Delta E / P_{\alpha}$, where $\Delta U=\hbar \omega / 4$ is the average energy deviation in the equivalent RLC circuit including the electromagnetic environment ${ }^{65}, \Delta E=e V-\hbar \omega$ is the energy barrier that the charge has to overcome during the transfer processes, and $P_{\alpha}=\left(\omega / 2 \pi+\left(\Gamma_{\alpha} / \Gamma_{\bar{\alpha}}-1\right) \mathrm{eV} / h\right)^{-1}$ is a period that characterizes the dynamics of the energy exchange associated to the reservoir $\alpha$ : the dynamics is faster and the heat noise higher (see Fig. 4(b)) in the strongest connected reservoir since one has $P_{\alpha}<P_{\bar{\alpha}}$ when $\Gamma_{\alpha}>\Gamma_{\bar{\alpha}}$. For symmetrical barriers, i.e., $a=1$, the period is identical in the two reservoirs and is equal to $2 \pi / \omega$, and the left and right heat noises coincide.

Experimentally, the heat noise at finite frequency could be obtained either from the measurement of the radiative power spectrum, as explained previously, or from the measurement of temperature fluctuations since one has the following relation 9110. $\mathcal{S}_{\alpha \alpha}^{\text {heat }}(\omega)=\left(1+\omega^{2} \tau_{E, \alpha}^{2}\right) K_{\alpha}^{2} \mathcal{S}_{\alpha \alpha}^{\text {temp }}(\omega)$, where $\mathcal{S}_{\alpha \alpha}^{\text {temp }}(\omega)=$ 

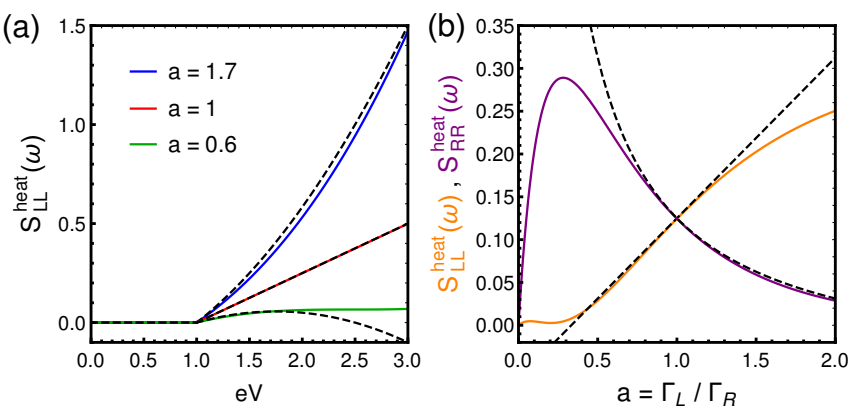

FIG. 4: Out-of-equilibrium (a) heat noise in the left reservoir as a function of voltage for three different values of the asymmetry factor $a=\Gamma_{L} / \Gamma_{R}$ with $\Gamma_{L}+\Gamma_{R}=40 \mathrm{meV}$. (b) Heat noise in the left and right reservoirs as a function of the asymmetry factor at $V=1.5 \mathrm{mV}$ and $\Gamma_{R}=10 \mathrm{meV}$. The other parameters are $\hbar \omega=1 \mathrm{meV}, k_{B} T_{L, R}=0.01 \mathrm{meV}$, and $\varepsilon_{0}=0$. The dashed lines correspond to Eq. (6).

$\int_{-\infty}^{\infty}\left\langle\Delta T_{\alpha}(t) \Delta T_{\alpha}(0)\right\rangle e^{-i \omega t} d t$. The energy relaxation time $\tau_{E, \alpha}$ is defined as the ratio between the heat capacity $C_{\alpha}=\partial\left\langle Q_{\alpha}\right\rangle / \partial T_{\alpha}$ and the thermal conductance $K_{\alpha}=$ $\partial\left\langle J_{\alpha}\right\rangle / \partial T_{\alpha}: \tau_{E, \alpha}=C_{\alpha} / K_{\alpha}$. When only electrons contribute to the heat current, one has $\tau_{E, \alpha}=\hbar \Gamma_{\alpha} /\left(\Gamma_{L} \Gamma_{R}\right)$.

\section{CONCLUSION}

The study of electronic heat noise reveals several features that are not visible in charge noise. At equilibrium and provided that $\sum_{\alpha} \Gamma_{\alpha} \lesssim 8 k_{B} T$, the Johnson-Nyquist heat noise represented as a function of the QD energy level $\varepsilon_{0}$ shows a double-peak structure instead of the single-peak structure visible in the charge noise. Out-of-equilibrium, since the QD can exchange energy with its electromagnetic environment, the heat noise does not vanish for perfect transmission, while the charge noise does, resulting in a crucial difference between these two quantities. Moreover, an out-of-equilibrium Planck's law is derived in that limit, meaning that the heat noise could be interpreted as the radiative power spectrum. Finally, unlike charge noise, heat noise is very sensitive to the coupling asymmetry, with a transition from a quadratic to a linear voltage variation when the couplings change from asymmetrical to symmetrical. A direct extension of this work would be the determination of the heat noise in an interacting QD using, for instance, the theory developed in Ref. 56 for the calculation of charge noise.

Acknowledgments - The author would like to acknowledge T.Q. Duong, P. Eyméoud, G. Fleury, J. Gabelli, M. Lavagna, F. Michelini, M. Moskalets, S. Sahoo and R. Zamoum for useful discussions.
${ }^{1}$ Z. Yu, G.-M. Tang, and J. Wang, Full-counting statistics of transient energy current in mesoscopic systems, Phys. Rev. B 93, 195419 (2016).

2 M. Silaev, T.T. Heikkilaä, and P. Virtanen, Lindbladequation approach for the full counting statistics of work and heat in driven quantum systems, Phys. Rev. E 90, 022103 (2014).

3 M. Kindermann and S. Pilgram, Statistics of heat transfer in mesoscopic circuits, Phys. Rev. B 69, 155334 (2004).

4 F. Battista, F. Haupt, and J. Splettstoesser, Energy and power fluctuations in ac-driven coherent conductors, Phys. Rev. B 90, 085418 (2014).

${ }^{5}$ M. Ridley, M. Galperin, E. Gull, and G. Cohen, Numerically exact full counting statistics of the energy current in the Kondo regime, Phys. Rev. B 100, 165127 (2019).

${ }^{6}$ F. Battista, M. Moskalets, M. Albert, and P. Samuelsson, Quantum Heat Fluctuations of Single-Particle Sources, Phys. Rev. Lett. 110, 126602 (2013).

7 T.T. Heikkilä and Y.V. Nazarov, Statistics of Temperature Fluctuations in an Electron System out of Equilibrium, Phys. Rev. Lett. 102, 130605 (2009).

8 T.L. van den Berg, F. Brange and P. Samuelsson, Energy and temperature fluctuations in the single electron box, New J. Phys. 17, 075012 (2015).

9 N. Dashti, M. Misiorny, P. Samuelsson, and J.
Splettstoesser, Probing charge- and heat-current noise by frequency-dependent fluctuations in temperature and potential, Phys. Rev. Appl. 10, 024007 (2018).

10 B. Karimi, F. Brange, P. Samuelsson, and J.P. Pekola, Reaching the ultimate energy resolution of a quantum detector, Nat. Commun. 11, 367 (2020).

11 M.A. Laakso, T.T. Heikkilä, and Y.V. Nazarov, Manifestly Non-Gaussian Fluctuations in SuperconductorNormal Metal Tunnel Nanostructures, Phys. Rev. Lett. 108, 067002 (2012).

12 R. Sánchez and M. Büttiker, Detection of single electron heat transfer statistics, Eur. Phys. Lett. 100, 47008 (2012).

13 M. Meschke, W. Guichard, and J.P. Pekola, Single-mode heat conduction by photons, Nature 444, 187 (2006).

14 A.V. Timofeev, M. Helle, M. Meschke, M. Möttönen, and J.P. Pekola, Electronic Refrigeration at the Quantum Limit, Phys. Rev. Lett. 102, 200801 (2009).

15 S. Ciliberto, A. Imparato, A. Naert, and M. Tanase, Heat Flux and Entropy Produced by Thermal Fluctuations, Phys. Rev. Lett. 110, 180601 (2013).

16 E. Sivre, H. Duprez, U. Gennser, and F. Pierre, Electronic heat flow and thermal shot noise in quantum circuits, Nat. Commun. 10, 5638 (2019).

17 S. Jezouin, F.D. Parmentier, A. Anthore, U. Gennser, A. Cavanna, Y. Jin, and F. Pierre, Quantum Limit of Heat 
Flow Across a Single Electronic Channel, Science 342, 601 (2013).

${ }^{18}$ K. Schwab, E.A. Henriksen, J.M. Worlock, and M.L. Roukes, Measurement of the quantum of thermal conductance, Nature 404, 974 (2000).

19 J.B. Pendry, Quantum limits to the flow of information and entropy, J. Phys. A: Math. Gen. 16, 2161 (1983).

20 E. Sivre, A. Anthore, F.D. Parmentier, A. Cavanna, U. Gennser, A. Ouerghi, Y. Jin, and F. Pierre, Heat Coulomb blockade of one ballistic channel, Nat. Phys. 14, 145 (2018).

21 D.V. Averin and J.P. Pekola, Violation of the FluctuationDissipation Theorem in Time-Dependent Mesoscopic Heat Transport, Phys. Rev. Lett. 104, 220601 (2010).

22 J.P. Pekola, and B. Karimi, Quantum Noise of ElectronPhonon Heat Current, J Low Temp Phys 191, 373 (2018).

23 A. Crépieux, Out-of-equilibrium fluctuation-dissipation relations verified by the electrical and thermoelectrical acconductances in a quantum dot, Ann. Phys. 529, 1600344 (2017).

${ }^{24}$ Y. Utsumi, O. Entin-Wohlman, A. Aharony, T. Kubo and Y. Tokura, Fluctuation theorem for heat transport probed by a thermal probe electrode, Phys. Rev. B 89, 205314 (2014).

25 C. Jarzynski, and D.K. Wójcik, Classical and Quantum Fluctuation Theorems for Heat Exchange, Phys. Rev. Lett. 92, 230602 (2004).

${ }^{26}$ M. Esposito, U. Harbola, and S. Mukamel, Nonequilibrium fluctuations, fluctuation theorems, and counting statistics in quantum systems, Rev. Mod. Phys. 81, 1665 (2009).

27 D.V. Averin and J.P. Pekola, Statistics of the dissipated energy in driven single-electron transitions, Eur. Phys. Lett. 96, 67004 (2011).

28 S. Rahav, U. Harbola, and S. Mukamel, Heat fluctuations and coherences in a quantum heat engine, Phy. Rev. A 86, 043843 (2012).

29 S Gasparinetti, P Solinas, A Braggio, and M Sassetti, Heat-exchange statistics in driven open quantum systems, New J. Phys. 16, 115001 (2014).

${ }^{30}$ F.W.J. Hekking and J.P. Pekola, Quantum Jump Approach for Work and Dissipation in a Two-Level System, Phys. Rev. Lett. 111, 093602 (2013).

31 M.P. Blencowe, Quantum energy flow in mesoscopic dielectric structures, Phys. Rev. B 59, 4992 (1999).

32 D. Sergi, Energy transport and fluctuations in small conductors, Phys. Rev. B 83, 033401 (2011).

33 A.A. Clerk, Full counting statistics of energy fluctuations in a driven quantum resonator, Phys. Rev. A 84, 043824 (2011).

34 F. Zhan, S. Denisov, and P. Hänggi, Electronic heat transport across a molecular wire: Power spectrum of heat fluctuations, Phy. Rev. B 84, 195117 (2011).

${ }^{35}$ F. Zhan, S. Denisov, and P. Hänggi, Power spectrum of electronic heat current fluctuations, Phys. Status Solidi B 250, 2355 (2013).

${ }^{36}$ I.V. Krive, E.N. Bogachek, A.G. Scherbakov, and U. Landman, Heat current fluctuations in quantum wires, Phys. Rev. B 64, 233304 (2001).

37 F. Ronetti, M. Acciai, D. Ferraro, J. Rech, T. Jonckheere, T. Martin, and Maura Sassetti, Symmetry Properties of Mixed and Heat Photo-Assisted Noise in the Quantum Hall Regime, Entropy 21, 730 (2019).

38 B.H. Wu and J.C. Cao, Phonon generation and phonon energy current fluctuation in QD molecules, J. Phys.: Con- dens. Matter 21, 245301 (2009).

39 B.K. Agarwalla, B. Li, and J.-S. Wang, Full-counting statistics of heat transport in harmonic junctions: Transient, steady states, and fluctuation theorems, Phy. Rev. E 85, 051142 (2012).

${ }^{40}$ R. Sánchez, B. Sothmann, A.N. Jordan, and M. Büttiker, Correlations of heat and charge currents in quantum-dot thermoelectric engines, New J. Phys. 15, 125001 (2013).

41 P. Eyméoud and A. Crépieux, Mixed electrical-heat noise spectrum in a QD, Phys. Rev. B 94, 205416 (2016).

42 R. Delagrange, J. Basset, H. Bouchiat, and R. Deblock, Emission noise and high frequency cut-off of the Kondo effect in a quantum dot, Phys. Rev. B 97, 041412(R) (2018).

43 G. Tang, Z. Yu, and J. Wang, Full-counting statistics of energy transport of molecular junctions in the polaronic regime, New J. Phys. 19, 083007 (2017).

44 B.K. Agarwalla, J.-H. Jiang, and D. Segal, Full counting statistics of vibrationally assisted electronic conduction: Transport and fluctuations of thermoelectric efficiency, Phys. Rev. B 92, 245418 (2015).

45 C. Wang, J. Ren, and J. Cao, Unifying quantum heat transfer in a nonequilibrium spin-boson model with full counting statistics, Phys. Rev. A 95, 023610 (2017).

46 H.M. Friedman, B.K. Agarwalla, and D. Segal, Quantum energy exchange and refrigeration: a full-counting statistics approach, New J. Phys. 20, 083026 (2018).

47 K. Saito and A. Dhar, Fluctuation Theorem in Quantum Heat Conduction, Phys. Rev. Lett. 99, 180601 (2007).

48 H.B. Callen, T.A. Welton, Irreversibility and Generalized Noise, Phys. Rev. 83, 34 (1951).

49 M. Moskalets, Floquet Scattering Matrix Theory of Heat Fluctuations in Dynamical Quantum Conductors, Phys. Rev. Lett. 112, 206801 (2014).

50 There exists an alternative definition for the heat current operator ${ }^{51}$, which could be of importance for timedependent transport. A recent discussion of this issue is presented in Ref. 52. However, it is claimed that it fails to describe the energy fluctuations 53 .

51 M.F. Ludovico, J.S. Lim, M. Moskalets, L. Arrachea, and D. Sánchez, Dynamical energy transfer in ac-driven quantum systems, Phys. Rev. B 89, 161306(R) (2014).

52 A.K. Slimane, P. Reck, and G. Fleury, Simulating timedependent thermoelectric transport in quantum systems, Phys. Rev. B 101, 235413 (2020).

53 M.A. Ochoa, A. Bruch, and A. Nitzan, Energy distribution and local fluctuations in strongly coupled open quantum systems: The extended resonant level model, Phys. Rev. B 94, 035420 (2016)

54 See Supplemental Material at https://journals.aps.org/prb /abstract/10.1103/PhysRevB.103.045427 for details of the calculation of the electronic heat noise in a quantum dot.

${ }^{55}$ R. Zamoum, M. Lavagna, and A. Crépieux, Nonsymmetrized noise in a QD: Interpretation in terms of energy transfer and coherent superposition of scattering paths, Phys. Rev. B 93, 235449 (2016).

56 A. Crépieux, S. Sahoo, T.Q. Duong, R. Zamoum, and M. Lavagna, Emission Noise in an Interacting Quantum Dot: Role of Inelastic Scattering and Asymmetric Coupling to the Reservoirs, Phys. Rev. Lett. 120, 107702 (2018)

57 A. Crépieux and F. Michelini, Mixed, charge and heat noises in thermoelectric nanosystems, J. Phys.: Condens. Matter 27, 015302 (2015).

58 M. Tsaousidou and G.P. Triberis, Thermoelectric proper- 
ties of a weakly coupled quantum dot: enhanced thermoelectric efficiency, J. Phys.: Condens. Matter 22, 355304 (2010).

59 P. Würfel, The chemical potential of radiation, J. Phys. C: Solid State Phys. 15, 3967 (1982).

60 P. Dawson, D.G. Walmsley, H.A. Quinn, and A.J.L. Ferguson, Observation and explanation of light-emission spectra from statistically rough $\mathrm{Cu}, \mathrm{Ag}$, and $\mathrm{Au}$ tunnel junctions, Phys. Rev. B 30, 3164 (1984).

61 R.D. Fedorovich, A.G. Naumovets, and P.M. Tomchuk, Electron and light emission from island metal films and generation of hot electrons in nanoparticles, Physics Reports 328, 73 (2000).

62 P. Février and J. Gabelli, Tunneling time probed by quan- tum shot noise, Nat. Commun. 9, 4940 (2018).

63 A. Martín-Jiménez, A.I. Fernández-Domínguez, K. Lauwaet, D. Granados, R. Miranda, F.J. GarcíaVidal, and R. Otero, Unveiling the radiative local density of optical states of a plasmonic nanocavity by STM, Nat. Commun. 11, 1021 (2020).

64 J. Basset, H. Bouchiat, and R. Deblock, Emission and Absorption Quantum Noise Measurement with an On-Chip Resonant Circuit, Phys. Rev. Lett. 105, 166801 (2010).

${ }^{65}$ Single Charge Tunneling: Coulomb blockade Phenomena in Nanostructures, edited by H. Grabert and M.H. Devoret, NATO ASI Series (Plenum, New-York, 1992). 